Article

\title{
Inverse Spectral Problems for Arbitrary-Order Differential Operators with Distribution Coefficients
}

\author{
Natalia P. Bondarenko ${ }^{1,2}$ iD \\ 1 Department of Applied Mathematics and Physics, Samara National Research University, \\ Moskovskoye Shosse 34, 443086 Samara, Russia; BondarenkoNP@info.sgu.ru \\ 2 Department of Mechanics and Mathematics, Saratov State University, Astrakhanskaya 83, \\ 410012 Saratov, Russia
}

check for updates

Citation: Bondarenko, N.P. Inverse Spectral Problems for

Arbitrary-Order Differential

Operators with Distribution

Coefficients. Mathematics 2021, 9 , 2989. https://doi.org/10.3390/ math9222989

Academic Editors: Miklós Rontó, András Rontó, Nino Partsvania, Bedřich Půža and Hriczó Krisztián

Received: 28 October 2021

Accepted: 18 November 2021

Published: 22 November 2021

Publisher's Note: MDPI stays neutral with regard to jurisdictional claims in published maps and institutional affiliations.

\section{Copyright: (c) 2021 by the authors.} Licensee MDPI, Basel, Switzerland. This article is an open access article distributed under the terms and conditions of the Creative Commons Attribution (CC BY) license (https:// creativecommons.org/licenses/by/ $4.0 /)$.
Abstract: In this paper, we propose an approach to inverse spectral problems for the $n$-th order $(n \geq 2)$ ordinary differential operators with distribution coefficients. The inverse problems which consist in the reconstruction of the differential expression coefficients by the Weyl matrix and by several spectra are studied. We prove the uniqueness of solution for these inverse problems, by developing the method of spectral mappings. The results of this paper generalize the previously known results for the second-order differential operators with singular potentials and for the higherorder differential operators with regular coefficients. In the future, the approach of this paper can be used for constructive solution and for investigation of solvability of the considered inverse problems.

Keywords: inverse spectral problems; higher-order differential operators; distribution coefficients; weyl matrix; uniqueness theorem

MSC: 34A55; 34B09; 34L05; 46F10

\section{Introduction}

In this paper, we study inverse spectral problems for the $n$-th order ordinary differential operators with distribution coefficients for $n \geq 2$. We consider the operators generated by the following differential expressions for $n=2 m$ :

$$
\begin{aligned}
\ell_{2 m}(y):=y^{(2 m)} & +\sum_{k=1}^{m}(-1)^{k}\left(\tau_{k}^{(k)}(x) y^{(m-k)}\right)^{(m-k)} \\
& +\mathrm{i} \sum_{k=1}^{m-1}(-1)^{k+1}\left(\left(\sigma_{k}^{(k)}(x) y^{(m-k-1)}\right)^{(m-k)}+\left(\sigma_{k}^{(k)}(x) y^{(m-k)}\right)^{(m-k-1)}\right),
\end{aligned}
$$

where

$$
\tau_{1}, \ldots, \tau_{m}, \sigma_{1}, \ldots, \sigma_{m-1} \in L_{2}(0,1)
$$

and for $n=2 m+1$ :

$$
\begin{aligned}
\ell_{2 m+1}(y):=y^{(2 m+1)} & +\mathrm{i} \sum_{k=1}^{m}(-1)^{k+1}\left(\tau_{k}^{(k)}(x) y^{(m-k)}\right)^{(m-k)} \\
& +\sum_{k=0}^{m-1}(-1)^{k}\left(\left(\sigma_{k}^{(k)}(x) y^{(m-k-1)}\right)^{(m-k)}+\left(\sigma_{k}^{(k)}(x) y^{(m-k)}\right)^{(m-k-1)}\right),
\end{aligned}
$$

where

$$
\tau_{1}, \ldots, \tau_{m}, \sigma_{0}, \ldots, \sigma_{m-1} \in L_{1}(0,1)
$$

The derivatives in (1) and (2) are understood in the sense of distributions. 
Note that, if the coefficients $\tau_{k}$ and $\sigma_{k}$ are sufficiently smooth, then the differential Expressions (1) and (2) can be easily reduced to the form

$$
y^{(n)}+\sum_{k=0}^{n-2} p_{k}(x) y^{(k)}, \quad n \geq 2,
$$

with some new coefficients $p_{k}(x), k=\overline{0, n-2}$. However, in the case of distribution coefficients, it is more natural to consider the higher-order differential expressions in the canonical forms (1) and (2), following Mirzoev and Shkalikov [1,2], who have developed a regularization approach to these operators. We also observe that, due to the well-known results (see, e.g., the book of Naimark [3]), every self-adjoint linear differential expression consists of the terms

$$
\left(p(x) y^{(v)}\right)^{(v)}, \quad \mathrm{i}\left[\left(p(x) y^{(v-1)}\right)^{(v)}+\left(p(x) y^{(v)}\right)^{(v-1)}\right],
$$

with real-valued coefficients $p(x)$. Anyway, in this paper, we study the general non-selfadjoint case, so the functions $\tau_{k}$ and $\sigma_{k}$ may be complex-valued.

Differential operators $\ell_{n}(y)$ of forms (1), (2) for certain values of $n$ arise in various applications. In particular, for $n=2$, operator (1) is equivalent (up to the sign) to the Sturm-Liouville operator

$$
-\ell_{2}(y)=-y^{\prime \prime}+q(x) y,
$$

which is used in classical mechanics for modeling string vibrations, in quantum mechanics for describing electron motion, and has other numerous applications.

For $n=3$, Expression (2) takes the form

$$
\mathrm{i} \ell_{3}(y)=\mathrm{i} y^{\prime \prime \prime}+\mathrm{i}(p(x) y)^{\prime}+q(x) y .
$$

The third-order operator (5) in the both self-adjoint and non-self-adjoint cases arises in the inverse problem method for integration of the nonlinear Boussinesq equation (see $[4,5]$ ). The spectral properties of the third-order differential operator have been studied in relation with mechanical problems of modeling thin membrane flow of viscous liquid and elastic beam vibrations (see [6] and references therein). In recent years, decreasing the smoothness of the differential operator coefficients causes interest of scholars. Spectral problems for the third-order operators (5) with non-smooth coefficients were investigated, e.g., in [6-8].

The fourth-order operator

$$
y^{(4)}+(p(x) y)^{\prime}+q(x) y,
$$

which is the special case of (1), is equivalent to the Euler-Bernoulli operator $\frac{1}{b(x)}\left(a(x) u^{\prime \prime}\right)^{\prime \prime}$ used for describing the beam vibrations (see $[9,10])$. Information about other applications of the fourth-order linear differential operators in mechanics, optics, and acoustics can be found in [11-13].

This paper is concerned with inverse spectral problems, which consist in the recovery of the differential operator coefficients from spectral characteristics. Such problems arise in quantum and classical mechanics, geophysics, meteorology, electronics, and other applications (see, e.g., [14] and references therein). The most complete results in the theory of inverse spectral problems have been obtained for the second-order Sturm-Liouville operator (4) with regular (integrable) potential $q(x)$ (see the monographs [14-17]).

In the last twenty years, spectral problems for the Sturm-Liouville operators with singular potentials $q \in W_{2}^{-1}(0,1)$ have been intensively studied. The class $W_{2}^{-1}(0,1)$ consists of functions $q=\sigma^{\prime}$, where $\sigma \in L_{2}(0,1)$ and the derivative is understood in the sense of distributions. This class contains, in particular, the Dirac $\delta$-function and the Coulumb potential $\frac{1}{x}$, which are used for modeling particle interactions in quantum mechanics [18]. 
Various approaches to the definition of the Sturm-Liouville operator with potential from $W_{2}^{-1}(0,1)$ were described by Savchuk and Shkalikov [19]. Hryniv and Mykytyuk [20-23] extended the transformation operator method to the inverse problems for the SturmLiouville operators with singular potentials and so generalized a number of classical results to this class of operators. Afterwards, the theory of inverse spectral problems for the second-order differential operators with singular potentials was developed in the studies of Savchuk and Shkalikov [24,25], Freiling et al. [26], Mykytyuk and Trush [27], Hryniv and Pronska [28,29], Eckhardt et al. [30], Guliyev [31], Bondarenko [32-34], etc.

Comparing with the second-order differential operators, the spectral theory of the higher-order differential operators of form (3) for $n>2$ is significantly more difficult for investigation. The classical transformation operator method, which was developed by Levitan [16] and Marchenko [15] and played an important role in the theory of the inverse Sturm-Liouville problems, appears to be ineffective for the higher-order operators. Consequently, relying on some ideas of Leibenson [35,36], Yurko [37,38] has created the method of spectral mappings. This method is based on the theory of analytic functions and, in particular, on the contour integration in the complex plane of the spectral parameter. By using the method of spectral mappings, Yurko $[37,38]$ has constructed the inverse problem theory for the higher-order operators (3) with regular coefficients on the half-line and on a finite interval and, later on, for many other classes of differential operators and pencils. Beals and his followers [39] developed another approach to the higher-order inverse scattering problems on the line.

In recent years, spectral theory of the higher-order differential operators with distribution coefficients is started to be investigated. Mirzoev and Shkalikov [1,2] have proposed an approach to the regularization of such operators. Savchuk and Shkalikov [40] have constructed the Birkhoff solutions with the certain asymptotic behavior as the spectral parameter tends to infinity for the differential equation of arbitrary even order with singular coefficients. However, as far as the author knows, there are still no results on inverse spectral problems for this class of operators. This paper aims to fill this gap and to start the investigation of the recovery of the higher-order differential operators with distribution coefficients from their spectral characteristics.

In this paper, we state the inverse spectral problem of recovering the coefficients $\left\{\tau_{k}\right\}$ and $\left\{\sigma_{k}\right\}$ of the differential equation $\ell_{n}(y)=\lambda y$, where $\ell_{n}(y)$ is defined by (1) or (2). As the initial data of the inverse problem, we use the Weyl matrix, which generalizes the Weyl functions for the second-order operators (see [14,15]). Weyl functions and their generalizations are natural spectral characteristics of inverse problem theory for various types of differential operators and pencils. It is worth mentioning that the Weyl matrix was first used by Yurko for the recovery of the regular higher-order differential operators (see $[37,38])$. Our main result is the uniqueness of the inverse problem solution (Theorem 2). As a corollary, we obtain the uniqueness of the reconstruction of the operator coefficients from several spectra of the equation $\ell_{n}(y)=\lambda y$ equipped with different boundary conditions. The proofs of the main results are based on the regularization of the differential expressions (1) and (2), on the use of the Birkhoff solutions, and on the development of the method of spectral mappings. In the future, our approach can be used for reduction of the nonlinear inverse problems to linear equations and for investigation of solvability for the studied inverse problems.

The paper is organized as follows: Sections 2 and 3 are preliminary. In Section 2, we describe the regularization of the differential expressions (1) and (2) from [1,2] and prove an auxiliary lemma related with this construction. In Section 3 , the equation $\ell_{n}(y)=\lambda y$ is reduced to the first-order system and the Birkhoff solutions are constructed. Section 3 is based on the results of $[40,41]$. In Section 4 , the equation $\ell_{n}(y)=\lambda y$ is equipped with the appropriate boundary conditions, the Weyl matrix is defined, and the inverse problem statements are provided. Furthermore, we investigate the relationship of the Weyl matrix with the several spectra. In Section 5, auxiliary asymptotic formulas are derived. In Section 6, we formulate and prove the main result, that is, the uniqueness theorem (Theorem 2) for the studied inverse problem. In Section 7 , we consider the examples of $n=2,3$, and compare 
the results of this paper with the previously known results for the second-order operators with singular potentials.

\section{Regularization}

In this section, we describe the regularization of the differential expression $\ell_{n}(y)$ obtained by Mirzoev and Shkalikov [1,2]. Furthermore, we prove an auxiliary lemma (Lemma 1), which will be important for the proofs of our main results.

Denote by $\Xi$ the collection of the coefficients $\Xi:=\left\{\tau_{1}, \ldots, \tau_{m}, \sigma_{i_{n}}, \ldots, \sigma_{m-1}\right\}$ of the expression $\ell_{n}(y)$ defined by (1) or (2), where $i_{n}=1$ if $n$ is even and $i_{n}=0$ if $n$ is odd. Given the coefficients $\Xi$, we define the matrix function $F(x)=\left[f_{k, j}(x)\right]_{k, j=1}^{n}$ constructed by the certain rule $F=\mathscr{F}(\Xi)$, which is described below.

Denote

$$
\varphi_{j}:=\tau_{j}+\mathrm{i} \sigma_{j-1}, \quad \psi_{j}:=\tau_{j}-\mathrm{i} \sigma_{j-1}, \quad j=\overline{1, m}, \quad\left(\sigma_{0}=0 \text { for even } n\right) .
$$

For $n=2 m$, the matrix $F=\mathscr{F}(\Xi)$ has been constructed in [1] and has the form

$$
F=\left[\begin{array}{cccccccccc}
0 & 1 & 0 & 0 & \ldots & 0 & 0 & \ldots & 0 & 0 \\
0 & 0 & 1 & 0 & \ldots & 0 & 0 & \ldots & 0 & 0 \\
\multicolumn{8}{c}{\ldots \ldots \ldots \ldots \ldots \ldots \ldots \ldots \ldots} \\
f_{m, 1} & f_{m, 2} & \ldots & f_{m, m} & 1 & 0 & \ldots & 0 & 0 \\
f_{m+1,1} & f_{m+1,2} & \ldots \ldots \ldots & f_{m+1, m+1} & 1 & \ldots & 0 & 0 \\
\multicolumn{8}{c}{\ldots \ldots \ldots \ldots \ldots \ldots \ldots \ldots} \\
f_{n-1,1} & f_{n-1,2} & \ldots \ldots & f_{n-1, m+1} & 0 & \ldots & 0 & 1 \\
f_{n, 1} & f_{n, 2} & \ldots \ldots & f_{n, m+1} & 0 & \ldots & 0 & 0
\end{array}\right],
$$

where

$$
\begin{aligned}
f_{k, j} & =0, \quad\left[\begin{array}{l}
k=\overline{1, m-1}, j=\overline{1, n}, \quad j \neq k+1, \\
k=\overline{m, n}, j=\overline{m+2, n},
\end{array}\right. \\
f_{k, k+1} & =1, \quad k=\overline{1, n-1}, \\
f_{m, m-j+1} & =(-1)^{j+1} \varphi_{j}, \quad f_{m+j, m+1}=-\psi_{j}, \quad j=\overline{1, m}, \\
f_{m+k, m-j} & =(-1)^{j+1} \varphi_{j+1} \psi_{k}+\chi_{j+k<m}(-1)^{j} C_{j+k+1}^{k}\left(\tau_{j+k+1}+\mathrm{i} \frac{j-k+1}{j+k+1} \sigma_{j+k}\right), \\
k & =\overline{1, m}, j=\overline{0, m-1}, \\
\chi_{j+k<m} & = \begin{cases}1, & \text { if } j+k<m, \quad C_{n}^{k}=\frac{n !}{k !(n-k) !} \text { are the binomial coefficients. }\end{cases}
\end{aligned}
$$

For $n=2 m+1$, the matrix $F=\mathscr{F}(\Xi)$ has the form (see [2]):

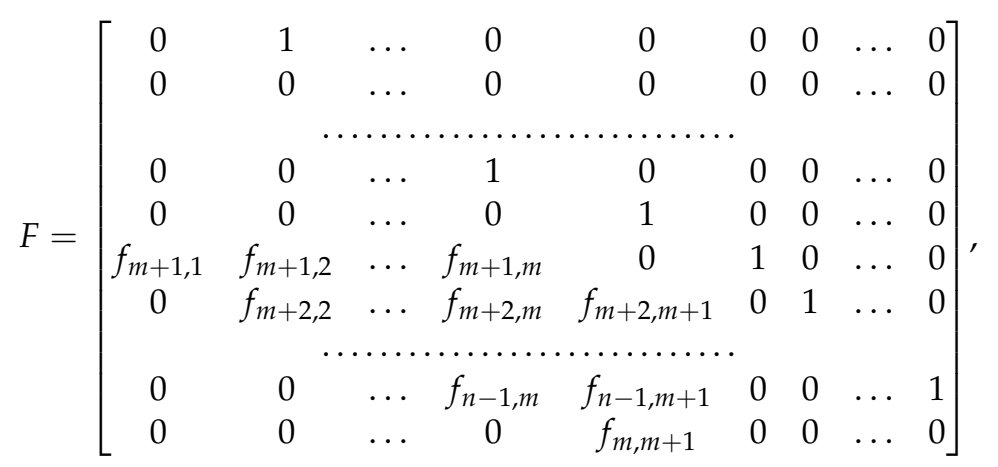


where

$$
\begin{aligned}
f_{k, j} & =0, \quad\left[\begin{array}{l}
k=\overline{1, m}, j=\overline{1, n}, \\
k=\overline{m+1, n}, j=\overline{m+2, n}, \quad j \neq k+1,
\end{array}\right. \\
f_{k, k+1} & =1, \quad k=\overline{1, n-1}, \quad f_{m+1, m+1}=0, \\
f_{m+1, m+1-j} & =(-1)^{j} \mathrm{i} \psi_{j}, \quad f_{m+j+1, m+1}=\mathrm{i} \varphi_{j}, \quad j=\overline{1, m}, \\
f_{m+k+1, m+k-j} & =(-1)^{j+k+1} C_{j+1}^{k}\left(\mathrm{i} \tau_{j+1}+\left(1-\frac{2 k}{j+1}\right) \sigma_{j}\right), \quad k=\overline{1, m-1}, \quad j=\overline{k, m-1} .
\end{aligned}
$$

Using the elements of the matrix function $F(x)$ defined by (6) or (9), introduce the quasi-derivatives

$$
y^{[0]}:=y, \quad y^{[k]}=\left(y^{[k-1]}\right)^{\prime}-\sum_{j=1}^{k} f_{k, j} y^{[j-1]}, \quad k=\overline{1, n} .
$$

Consider the operator $\mathcal{F} y:=y^{[n]}$ with the domain

$$
\mathcal{D}(\mathcal{F})=\left\{y: y^{[k]} \in A C[0,1], k=\overline{0, n-1}\right\} .
$$

Obviously, the operator $\mathcal{F}$ is correctly defined in $L_{1}(0,1)$. Moreover, it has been shown in $[1,2]$ that, for $y \in \mathcal{D}(\mathcal{F})$, the relation $\ell_{n}(y)=\mathcal{F} y$ holds in the distribution space $\mathfrak{D}^{\prime}(0,1)$. $\left(\mathfrak{D}^{\prime}(0,1)\right.$ is the space of continuous linear functionals on $\left.\mathfrak{D}(0,1)=C_{0}^{\infty}(0,1)\right)$.

Note that, in $[1,2]$, the construction of the matrix $F(x)$ was given for differential expressions of a more general form than $l_{n}(y)$. In this paper, we confine ourselves to the case with the coefficient 1 at $y^{(n)}$ and the coefficient 0 at $y^{(n-1)}$. This case is natural for investigation of inverse spectral problems.

For study of inverse problems, we need the following lemma.

Lemma 1. Suppose that $F=\mathscr{F}(\Xi), \tilde{F}=\mathscr{F}(\tilde{\Xi})$ and a unit lower-triangular matrix function $P(x)=\left[p_{k, j}(x)\right]_{k, j=1}^{n}$ satisfies

$$
P^{\prime}(x)+P(x) \tilde{F}(x)=F(x) P(x), \quad x \in(0,1), \quad P(0)=I .
$$

Then, $P(x) \equiv I$ and $F(x)=\tilde{F}(x)$ a.e. on $(0,1)$.

Proof. For brevity, we omit the argument $(x)$ of $p_{k, j}(x)$ and $f_{k, j}(x)$ in this proof. It is given that $p_{k, j}=0$ for $k<j$ and $p_{k, k}=1$ for $k=\overline{1, n}$.

Case 1: $n=2 m$. Let us show that $p_{k, j}=0$ for all $k=\overline{1, m}$ and $j<k$ by induction. For $k=1$, the assertion is trivial. For $k<m$ and $j \leq k$, relation (10) in the element-wise form yields

$$
p_{k, j}^{\prime}+p_{k, j-1}=p_{k+1, j} .
$$

Here, and below, we assume that $p_{k, j}=0$ if $j<1$ or $k>n$. If we have already proved the assertion $p_{k, j}=0$ for $j<k$ in the $k$-th row, then the left-hand side of (11) equals zero, so we immediately get the assertion for the $(k+1)$-th row. Using the $m$-th row of (10), we obtain

$$
p_{m+1, j}=\tilde{f}_{m, j}-f_{m, j}, \quad j=\overline{1, m} .
$$

Similarly, considering the $j$-th column of (10) for $j=n, n-1, \ldots, m+1$, we get $p_{k, j}=0$ for all $k>j, j=\overline{m+1, n}$, and

$$
p_{k, m}=f_{k, m+1}-\tilde{f}_{k, m+1}, \quad k=\overline{m+1, n} .
$$

Note that there is no contradiction between (12) and (13), since 


$$
p_{m+1, m}=\tilde{\varphi}_{1}-\varphi_{1}=\tilde{\psi}_{1}-\psi_{1}
$$

and $\varphi_{1}=\psi_{1}=\tau_{1}$ for even $n$.

Let us prove that $p_{k, j}=0$ for $k=\overline{m+1, n}, j=\overline{1, m}$. We will prove this assertion for all such $k, j$ that $k-j=d$ by iterating over the values of $d$ in the following order: $d=n-1, n-2, \ldots, 2,1$. Consider some fixed $d$. Suppose that, for all the larger values of $d$, the assertion is already proved. Using (10) and taking the obtained relations into account, we derive

$$
p_{k, j}^{\prime}+p_{k, j-1}+p_{k, m} \tilde{f}_{m, j}+\tilde{f}_{k, j}=f_{k, j}+f_{k, m+1} p_{m+1, j}+p_{k+1, j,} \quad k=\overline{m+1, n}, \quad j=\overline{1, m} .
$$

We have already proved that $p_{k, j-1}=p_{k+1, j}=0$. Consequently, using (12) and (13), we simplify (14):

$$
p_{k, j}^{\prime}+\tilde{f}_{k, j}-\tilde{f}_{k, m+1} \tilde{f}_{m, j}=f_{k, j}-f_{k, m+1} f_{m, j}
$$

It follows from (7) and (8) that

$$
f_{k, j}-f_{k, m+1} f_{m, j}=\chi_{d<m} C_{k-j+1}^{k-m}\left(\tau_{d+1}+\mathrm{i} \frac{2 m-j-k}{k-j+1} \sigma_{d}\right), \quad k=\overline{m+1, n}, \quad j=\overline{1, m} .
$$

If $d \geq m$, then (15) and (16) readily imply $p_{k, j}^{\prime}=0$. Consider the case $d<m$. In this case, we have already proved that $p_{m+1, m-d}=0$ and $p_{m+d+1, m}=0$. By virtue of (7), (12) and (13), this yields $\tau_{d+1}=\tilde{\tau}_{d+1}, \sigma_{d}=\tilde{\sigma}_{d}$. Hence, we have

$$
f_{k, j}-f_{k, m+1} f_{m, j}=\tilde{f}_{k, j}-\tilde{f}_{k, m+1} \tilde{f}_{m, j}
$$

so (15) implies $p_{k, j}^{\prime}=0$. Taking the initial condition $P(0)=I$ into account, we conclude that $p_{k, j}=0$. Thus, the assertion is proved for $k-j=d$. By induction, it is valid for all $k=\overline{m+1, n}, j=\overline{1, n}$. This concludes the proof for even $n$.

Case 2: $n=2 m+1$. Similarly to the case of even $n$, one can easily show that $p_{k, j}=0$ for $k<j$ and $(k=\overline{1, m}$ or $j=\overline{m, n})$. It remains to prove that $p_{k, j}=0$ for $k=\overline{m+1, n}$, $j=\overline{1, m-1}$. Consider the rows in the order $k=n, n-1, \ldots, m+1$. For each fixed row, consider the columns in the order $j=1,2, \ldots, m-1$. Using (10) in the element-wise form and taking the special structure of the matrix (9) into account, we obtain

$$
p_{k, j}^{\prime}+p_{k, j-1}=p_{k+1, j}
$$

If the assertion is supposed to be proved for all the previous values of $(k, j)$, then $p_{k, j-1}=p_{k+1, j}=0$. Hence, $p_{k, j}^{\prime}=0$, which implies $p_{k, j}=0$. This yields the claim of the lemma.

\section{Birkhoff Solutions}

In this section, we briefly describe the construction of the Birkhoff solutions with the certain behavior as $|\rho| \rightarrow \infty$ for the higher-order differential equation $\ell_{n}(y)=\rho^{n} y$ with distribution coefficients. This construction is based on the reduction of the considered equation to a first-order system of form (20). For even order $n$, the Birkhoff solution have been obtained in [40]. For odd $n$, the reduction is similar. For obtaining the Birkhoff solutions of the first-order system, the results of either [40] or [41] can be applied.

Let $l_{n}(y)$ be the differential expression defined by (1) or (2) with the coefficients $\Xi$, and let $F=\mathscr{F}(\Xi)$. For a function $y \in \mathcal{D}(\mathcal{F})$, denote the column vector of its quasiderivatives by

$$
\vec{y}(x)=\operatorname{col}\left(y^{[0]}(x), y^{[1]}(x), \ldots, y^{[n-1]}(x)\right) .
$$

We say that $y$ is a solution of the equation

$$
\ell_{n} y=\rho^{n} y, \quad x \in(0,1),
$$


if $y \in \mathcal{D}(\mathcal{F})$ and

$$
\vec{y}^{\prime}=(F(x)+\Lambda) \vec{y}, \quad x \in(0,1),
$$

where $\rho \in \mathbb{C}, \Lambda:=\rho^{n} E_{n, 1}$. Here, and below, $E_{k, j}$ is the $(n \times n)$ matrix whose element at the position $(k, j)$ equals 1 and all the other elements equal zero. It can be shown that, if $y \in \mathcal{D}(\mathcal{F})$ and $\vec{y}$ satisfies (18), then (17) holds in $\mathfrak{D}^{\prime}(0,1)$.

In view of (6) and (9), the change of variables $\vec{y}(x)=\operatorname{diag}\left\{1, \rho, \ldots, \rho^{n-1}\right\} u(x)$ transforms the system (18) into

$$
u^{\prime}(x)=F(x, \rho) u
$$

where

$$
\begin{gathered}
F(x, \rho)=\rho F_{1}+F_{0}(x)+\sum_{k=1}^{n-1} \rho^{-k} F_{-k}(x), \\
F_{1}=\left[\begin{array}{cccccc}
0 & 1 & 0 & \ldots & 0 & 0 \\
0 & 0 & 1 & \ldots & 0 & 0 \\
& \ldots \ldots \ldots & \ldots & \\
0 & 0 & 0 & \ldots & 1 & 0 \\
0 & 0 & 0 & \ldots & 0 & 1 \\
1 & 0 & 0 & \ldots & 0 & 0
\end{array}\right], \\
F_{0}(x)= \begin{cases}\tau_{1}(x)\left(E_{m, m}-E_{m+1, m+1}\right), & n=2 m, \\
0, & n=2 m+1,\end{cases}
\end{gathered}
$$

and $F_{-k} \in L_{1}(0,1), k=\overline{1, n-1}$.

Denote by $\left\{\omega_{j}\right\}_{j=1}^{n}$ the roots of the equation $\omega^{n}=1$ numbered in some fixed order. Put $B:=\operatorname{diag}\left\{\omega_{1}, \omega_{2}, \ldots, \omega_{n}\right\}, \Omega:=\left[\omega_{k}^{j-1}\right]_{j, k=1}^{n}$. Obviously, $\Omega^{-1} F_{1} \Omega=B$. Consequently, the system (19) can be reduced to the form

$$
\begin{aligned}
v^{\prime} & =\rho B v+A(x) v+C(x, \rho) v, \\
v(x)=\Omega^{-1} u(x), \quad A(x) & =\Omega^{-1} F_{0}(x) \Omega, \quad C(x, \rho)=\sum_{k=1}^{n-1} \rho^{-k} \Omega^{-1} F_{-k}(x, \rho) \Omega .
\end{aligned}
$$
odd $n$.

Observe that the elements of $A(x)$ belong to $L_{2}(0,1)$ for even $n$ and $A(x) \equiv 0$ for

The Birkhoff solutions for systems of more general forms than (20) have been constructed in $[40,41]$. Those results readily imply the Birkhoff solutions of Equation (17).

Consider the partition of the $\rho$-plane into the sectors

$$
\Gamma_{k}=\left\{\rho: \frac{\pi(k-1)}{n}<\arg \rho<\frac{\pi k}{n}\right\}, \quad k=\overline{1,2 n} .
$$

Below, we assume that, if $\rho$ lies in a fixed sector $\Gamma=\Gamma_{k}$, then the roots of the equation $\omega^{n}=1$ are numbered so that

$$
\operatorname{Re}\left(\rho \omega_{1}\right)<\operatorname{Re}\left(\rho \omega_{2}\right)<\cdots<\operatorname{Re}\left(\rho \omega_{n}\right), \quad \rho \in \Gamma .
$$

Proposition 1. For each fixed sector $\Gamma$ and some $\rho^{*}>0$, Equation (17) has a fundamental system of solutions (FSS) $\left\{y_{k}(x, \rho)\right\}_{k=1}^{n}$ such that the quasi-derivatives $y_{k}^{[j]}(x, \rho)$ are analytic in $\rho$ for each fixed $x \in[0,1], \rho \in \Gamma,|\rho| \geq \rho^{*}$, and

$$
y_{k}^{[j]}(x, \rho)=\left(\rho \omega_{k}\right)^{j} \exp \left(\rho \omega_{k} x\right)(1+o(1)), \quad|\rho| \rightarrow \infty, \quad \rho \in \bar{\Gamma},
$$

uniformly with respect to $x \in[0,1], j=\overline{0, n-1}, k=\overline{1, n}$. 
In [40], the Birkhoff solutions $\left\{y_{k}(x, \rho)\right\}_{k=1}^{n}$ have been constructed for even $n$, and their properties have been studied in more detail. In particular, the behavior of the remainder term in (23) was studied in various spaces. The case of the odd $n$ can be analyzed similarly. It can be shown that, since $A(x) \equiv 0$ for odd $n$, then the remainder term is $O\left(\rho^{-1}\right)$ in this case. However, the estimate $o(1)$ is sufficient for the purposes of the present paper, so we do not elaborate into details.

\section{Inverse Problems}

The goals of this section are to define the spectral characteristics of the operators induced by the differential equation $\ell_{n}(y)=\lambda y$ and to formulate the inverse spectral problems. First, we introduce the boundary conditions by using the quasi-derivatives. Second, the special FSS of Equation (17) generated by certain boundary conditions are defined. We also define the Weyl matrix, which is used as the main spectral characteristics. Further in this section, we establish the connection between the Weyl matrix and the several spectra. Finally, the inverse problems are formulated.

For $y \in \mathcal{D}(\mathcal{F})$, define the linear forms

$$
\mathcal{U}_{\tilde{\xi} a}(y)=U_{\tilde{\xi} a} \vec{y}(a), \quad \xi=\overline{1, n}, \quad a=0,1,
$$

where $U_{\xi_{a} a}$ are row vectors of length $n$. For $a=0,1$, denote by $U_{a}$ the matrix whose $\xi$-th row is $U_{\xi a}, \xi=\overline{1, n}$. Assume that $U_{a}=P_{a} L_{a}$, where $P_{a}$ is a permutation matrix and $L_{a}$ is a unit lower triangular matrix. This means that the matrix $P_{a}$ has the elements equal to 1 at the positions $\left(k, p_{k, a}+1\right), k=\overline{1, n}$, where $\left\{\left(p_{k, a}+1\right)\right\}_{k=1}^{n}$ is the permutation of the numbers $\{1,2, \ldots, n\}$, and all the other elements are zero. The elements of $L_{a}$ satisfy $l_{j j}=1$, $l_{j k}=0$ for $j<k$. In other words,

$$
\mathcal{U}_{\tilde{\zeta} a}(y)=y^{\left[p_{\tilde{\xi}, a}\right]}(a)+\sum_{\nu=0}^{p_{\tilde{\xi}, a}-1} u_{\tilde{\xi} v a} y^{[v]}(a), \quad \xi=\overline{1, n}, \quad a=0,1,
$$

so these linear forms are analogous to the ones considered in ([38], Section 2).

Put $\lambda=\rho^{n}$. Denote by $\left\{C_{k}(x, \lambda)\right\}_{k=1}^{n}$ the solutions of Equation (17) satisfying the initial conditions

$$
\mathcal{U}_{\xi 0}\left(C_{k}\right)=\delta_{\xi k}, \quad \xi=\overline{1, n},
$$

where $\delta_{\xi k}$ is the Kronecker delta. Equivalently, the matrix function $C(x, \lambda)=\left[\vec{C}_{k}(x, \lambda)\right]_{k=1}^{n}$ satisfies Equation (18) and the initial condition

$$
C(0, \lambda)=U_{0}^{-1} \text {. }
$$

Note that, since $U_{0}=P_{0} L_{0}$, then $\operatorname{det} U_{0}= \pm 1$. Clearly, the quasi-derivatives $C_{k}^{[j]}(x, \lambda)$, $k=\overline{1, n}, j=\overline{0, n-1}$, are absolutely continuous on $[0,1]$ for each fixed $\lambda$ and entire in $\lambda$ for each fixed $x \in[0,1]$.

Denote by $\left\{\Phi_{k}(x, \lambda)\right\}_{k=1}^{n}$ the solutions of Equation (17) satisfying the boundary conditions

$$
\mathcal{U}_{\xi 0}\left(\Phi_{k}\right)=\delta_{\xi k}, \quad \xi=\overline{1, k}, \quad \mathcal{U}_{\eta 1}\left(\Phi_{k}\right)=0, \quad \eta=\overline{k+1, n} .
$$

Equivalently, the vector functions $\left\{\vec{\Phi}_{k}(x, \lambda)\right\}_{k=1}^{n}$ satisfy Equation (18) and the boundary conditions

$$
U_{\xi 0} \vec{\Phi}_{k}(0, \lambda)=\delta_{\xi k}, \quad \xi=\overline{1, k}, \quad U_{\eta 1} \vec{\Phi}_{k}(1, \lambda)=0, \quad \eta=\overline{k+1, n} .
$$

The existence of such solutions $\left\{\Phi_{k}(x, \lambda)\right\}_{k=1}^{n}$ follows from the arguments below. 
Consider the matrix function $\Phi(x, \lambda)=\left[\vec{\Phi}_{k}(x, \lambda)\right]_{k=1}^{n}$. Since the columns of the matrix $C(x, \lambda)$ form a FSS of the system (18), then

$$
\Phi(x, \lambda)=C(x, \lambda) M(\lambda),
$$

where $M(\lambda)=\left[M_{j k}(\lambda)\right]_{j, k=1}^{n}$ is called the Weyl matrix. The first row of the matrix relation (28) has the form

$$
\Phi_{k}(x, \lambda)=\sum_{j=1}^{n} M_{j k}(\lambda) C_{j}(x, \lambda), \quad k=\overline{1, n} .
$$

Using (24), (26) and (29), we show that

$$
\begin{aligned}
M_{\tilde{\xi} k}(\lambda)=\delta_{\xi k}, \quad \xi & =\overline{1, k}, \\
\mathcal{U}_{\eta 1}\left(C_{k}\right)+\sum_{j=k+1}^{n} M_{j k}(\lambda) \mathcal{U}_{\eta 1}\left(C_{j}\right) & =0, \quad \eta=\overline{k+1, n} .
\end{aligned}
$$

By virtue of (30), the Weyl matrix has the lower-triangular form

$$
M(\lambda)=\left[\begin{array}{ccccc}
1 & 0 & 0 & \ldots & 0 \\
M_{21}(\lambda) & 1 & 0 & \ldots & 0 \\
M_{31}(\lambda) & M_{32}(\lambda) & 1 & \ldots & 0 \\
& \ldots \ldots \ldots \ldots \ldots \ldots & & \\
M_{n 1}(\lambda) & M_{n 2}(\lambda) & M_{n 3}(\lambda) & \ldots & 1
\end{array}\right]
$$

For each fixed $k=\overline{1, n-1}$, relations (31) can be treated as the system of linear equations with respect to $\left[M_{j k}(\lambda)\right]_{j=k+1}^{n}$. Solving this system by Cramer's rule, we find

$$
M_{j k}(\lambda)=-\frac{\Delta_{j k}(\lambda)}{\Delta_{k k}(\lambda)}, \quad k=\overline{1, n-1}, \quad j=\overline{k+1, n},
$$

where

$$
\Delta_{k k}(\lambda):=\left|\begin{array}{ccc}
\mathcal{U}_{k+1,1}\left(C_{k+1}\right) & \ldots & \mathcal{U}_{k+1,1}\left(C_{n}\right) \\
\ldots \ldots \ldots \ldots \ldots & \ldots \ldots \\
\mathcal{U}_{n 1}\left(C_{k+1}\right) & \ldots & \mathcal{U}_{n 1}\left(C_{n}\right)
\end{array}\right|,
$$

and $\Delta_{j k}(\lambda)$ is obtained from $\Delta_{k k}(\lambda)$ by the replacement of $C_{j}$ by $C_{k}$. Equivalently,

$$
\Delta_{k k}(\lambda)=\operatorname{det}\left(T_{k}+T_{k}^{\perp} U_{1} C(1, \lambda)\right), \quad \Delta_{j k}(\lambda)=\operatorname{det}\left(T_{k}+T_{k}^{\perp} U_{1} C(1, \lambda) P_{j k}\right),
$$

where

$$
T_{k}=\left[T_{k, l s}\right]_{l, s=1}^{n}, \quad T_{k, l s}=\left\{\begin{array}{ll}
1, & l=s \leq k, \\
0, & \text { otherwise, }
\end{array} \quad T_{k}^{\perp}=I-T_{k},\right.
$$

and the matrix $P_{j k}$ is obtained from the unit matrix by permutation of the $j$-th and the $k$-th columns.

Thus, one can construct the elements of the Weyl matrix $M(\lambda)$ by formulas (30), (32) and (33) and then find the matrix function $\Phi(x, \lambda)$ by (28). The elements $\left\{\Phi_{k}(x, \lambda)\right\}_{k=1}^{n}$ of the first row will satisfy Equation (17) and the boundary conditions (26). Therefore, the solutions $\left\{\Phi_{k}(x, \lambda)\right\}_{k=1}^{n}$ are correctly defined for such $\lambda$ that $\Delta_{k k}(\lambda) \neq 0, k=\overline{1, n-1}$.

It follows (33) that the functions $\Delta_{j k}(\lambda)$ are entire in $\lambda$. It will be shown in the next section that, for $k=\overline{1, n-1}, j=\overline{k, n}$, we have $\Delta_{j k}(\lambda) \not \equiv 0$ and the set of zeros of $\Delta_{j k}(\lambda)$ is countable. Consequently, the matrix functions $M(\lambda)$ and $\Phi(x, \lambda)$ for each fixed $x \in[0,1]$ are meromorphic in $\lambda$.

Remark 1. Note that the row vector $U_{11}$ does not participate in the definitions of the Weyl solution and the Weyl matrix. This row is added to the coefficient matrix $U_{1}$ only for convenience and can 
be arbitrary. Furthermore, in view of (27), the vector $U_{\eta 1}$ can be replaced by $U_{\eta 1}-c U_{\theta 1}$ with arbitrary $c \in \mathbb{C}$ if $\eta<\theta$ and $p_{\eta, 1}>p_{\theta, 1}$. This replacement does not change the Weyl solution $\Phi(x, \lambda)$. Therefore, the matrix $U_{1}$ is not uniquely determined by $\Phi(x, \lambda)$.

Theorem 1. For each fixed pair $(j, k)$ such that $1 \leq k \leq j \leq n$, the zeros $\left\{\lambda_{s, j k}\right\}_{s \geq 1}$ of the function $\Delta_{j k}(\lambda)$ coincide with the eigenvalues of the boundary value problem

$$
\mathcal{L}_{j k}: \quad \ell_{n}(y)=\lambda y, \quad \mathcal{U}_{\xi 0}(y)=0, \quad \xi=\overline{1, k-1}, j, \quad \mathcal{U}_{\eta 1}(y)=0, \quad \eta=\overline{k+1, m} .
$$

Proof. Let $\lambda_{0}$ be an eigenvalue of $\mathcal{L}_{j k}$ and $y(x)$ be the corresponding eigenfunction. Then,

$$
y(x)=\sum_{l=1}^{n} \alpha_{l} C_{l}\left(x, \lambda_{0}\right)
$$

Substituting this expansion into the boundary conditions of $\mathcal{L}_{j k}$, we obtain the linear system

$$
\begin{aligned}
& \sum_{l=1}^{n} \alpha_{l} \mathcal{U}_{\xi 0}\left(C_{l}\left(x, \lambda_{0}\right)\right)=0, \quad \xi=\overline{1, k-1}, j, \\
& \sum_{l=1}^{n} \alpha_{l} \mathcal{U}_{\eta 1}\left(C_{l}\left(x, \lambda_{0}\right)\right)=0, \quad \eta=\overline{k+1, n},
\end{aligned}
$$

with respect to $\left\{\alpha_{l}\right\}_{l=1}^{n}$. In view of (24), the determinant of the obtained system equals $\Delta_{j k}\left(\lambda_{0}\right)$ (up to the sign). Hence, a non-trivial solution $y(x)$ of $\mathcal{L}_{j k}$ exists if and only if $\Delta_{j k}\left(\lambda_{0}\right)=0$. This yields the claim.

Consider the following inverse problems:

Inverse Problem 4.1. Given the Weyl matrix $M(\lambda)$, find $\Xi$.

Inverse Problem 4.2. Given the eigenvalues $\left\{\lambda_{s, j k}\right\}_{s \geq 1}, k=\overline{1, n-1}, j=\overline{k, n}$, find $\Xi$.

It is shown below (see Remark 2) that the entire functions $\Delta_{j k}(\lambda)$ have the order $\frac{1}{n}$. Therefore, by Hadamard's factorization theorem, these functions can be constructed by their zeros as infinite products:

$$
\Delta_{j k}(\lambda)=C_{j k} \prod_{s=1}^{\infty}\left(1-\frac{\lambda}{\lambda_{s, j k}}\right), \quad 1 \leq k \leq j \leq n,
$$

if $\lambda_{s, j k} \neq 0$. The case $\lambda_{s, j k}=0$ requires minor technical changes. The constants $C_{j k} \neq 0$ can be easily found by using the asymptotics of Lemma 2 . Thus, given the eigenvalues $\left\{\lambda_{s, j k}\right\}_{s \geq 1}$, one can find the characteristic functions $\Delta_{j k}(\lambda)$ and then construct all the nontrivial elements of the Weyl matrix by (32). In this way, Inverse Problem 4.2 is reduced to Inverse Problem 4.1. Therefore, we further focus on Inverse Problem 4.1.

Note that it is possible to determine the functions $\tau_{k}$ and $\sigma_{k}$ (not only their derivatives $\tau_{k}^{(k)}$ and $\sigma_{k}^{(k)}$ ) because the boundary conditions contain the quasi-derivatives. We assume that the matrices $U_{0}$ and $U_{1}$, defining the boundary conditions, are known a priori. Otherwise, it may be impossible to determine $U_{0}$ and $U_{1}$ from the Weyl matrix (see Remark 1 and Example 1).

\section{Asymptotics}

In this section, we obtain auxiliary results concerning the asymptotic behavior of the characteristic functions $\Delta_{j k}(\lambda)$ and of the solutions of Equation (17). For this purpose, the solutions $\left\{C_{k}\right\}_{k=1}^{n}$ and $\left\{\Phi_{k}\right\}_{k=1}^{n}$ are expanded over the Birkhoff FSS $\left\{y_{k}\right\}_{k=1}^{n}$ from Proposition 1. 


\section{Denote}

$$
\begin{gathered}
r_{k}:=\sum_{j=1}^{k} p_{j, 0}+\sum_{j=k+1}^{n} p_{j, 1}, \quad m_{k}:=r_{k}-\frac{n(n-1)}{2}, \quad s_{k}=\sum_{j=k+1}^{n} \omega_{j}, \\
\Theta(\rho):=\operatorname{diag}\left\{1, \rho, \ldots, \rho^{n-1}\right\}, \quad E(x, \rho):=\operatorname{diag}\left\{\exp \left(\rho \omega_{k} x\right)\right\}_{k=1^{\prime}}^{n} \\
{[1]=1+o(1), \quad[I]=I+o(1), \quad|\rho| \rightarrow \infty .}
\end{gathered}
$$

In Lemmas 2 and 3, we suppose that $\Gamma$ is a fixed sector of form (21), the roots $\left\{\omega_{j}\right\}_{j=1}^{n}$ of the equation $\omega^{n}=1$ are numbered according to (22), and $\{\rho: \arg \rho=\varphi\} \subset \Gamma$ is a fixed ray. For a fixed sector $\Gamma$, we define the matrix function $Y(x, \rho)=\left[\vec{y}_{k}(x, \rho)\right]_{k=1}^{n}$, where $\left\{y_{k}(x, \rho)\right\}_{k=1}^{n}$ is the Birkhoff FSS described in Proposition 1. The asymptotics (23) imply

$$
Y(x, \rho)=\Theta(\rho) \Omega[I] E(x, \rho), \quad|\rho| \rightarrow \infty, \quad \rho \in \bar{\Gamma} .
$$

Lemma 2. The following asymptotic relation holds as $|\rho| \rightarrow \infty, \arg \rho=\varphi$ :

$$
\Delta_{j k}(\lambda)=c_{j k} \rho^{m_{k}-p_{k, 0}+p_{j, 0}} \exp \left(\rho s_{k}\right)[1], \quad c_{j k} \neq 0, \quad 1 \leq k \leq j \leq n,
$$

Proof. The matrix function $C(x, \lambda)$ can be represented as

$$
C(x, \lambda)=Y(x, \rho) \mathcal{A}(\rho),
$$

where $\lambda=\rho^{n}, \rho \in \bar{\Gamma},|\rho| \geq \rho^{*}, \mathcal{A}(\rho)$ is an $(n \times n)$ matrix. Using (25), (36) and (38), we obtain

$$
\mathcal{A}(\rho)=[I] \Omega^{-1}(\Theta(\rho))^{-1} U_{0}^{-1}, \quad|\rho| \rightarrow \infty, \quad \rho \in \bar{\Gamma} .
$$

Hence,

$$
\operatorname{det} \mathcal{A}(\rho)=c_{\mathcal{A}} \rho^{-n(n-1) / 2}[1], \quad|\rho| \rightarrow \infty, \quad \rho \in \bar{\Gamma}, \quad c_{\mathcal{A}} \neq 0 .
$$

Relations (25) and (33) imply

$$
\Delta_{k k}(\lambda)=\operatorname{det}\left(T_{k} U_{0} C(0, \lambda)+T_{k}^{\perp} U_{1} C(1, \lambda)\right), \quad k=\overline{1, n-1} .
$$

Substitution of (38) into (40) yields

$$
\Delta_{k k}(\lambda)=d_{k}(\rho) \operatorname{det} \mathcal{A}(\rho)
$$

where

$$
d_{k}(\rho):=\operatorname{det}\left(T_{k} U_{0} Y(0, \rho)+T_{k}^{\perp} U_{1} Y(1, \rho)\right) .
$$

Recall that $U_{a}=P_{a} L_{a}, a=0,1$. For the unit lower triangular matrix $L_{a}$, we have

$$
L_{a} \Theta(\rho)=\Theta(\rho)\left(I+O\left(\rho^{-1}\right)\right) .
$$

Consequently, taking (36) into account, we obtain

$$
\begin{aligned}
& T_{k} U_{0} Y(0, \rho)+T_{k}^{\perp} U_{1} Y(1, \rho)=T_{k} P_{0} \Theta(\rho) \Omega[I]+T_{k}^{\perp} P_{1} \Theta(\rho) \Omega[I] E(1, \rho),
\end{aligned}
$$

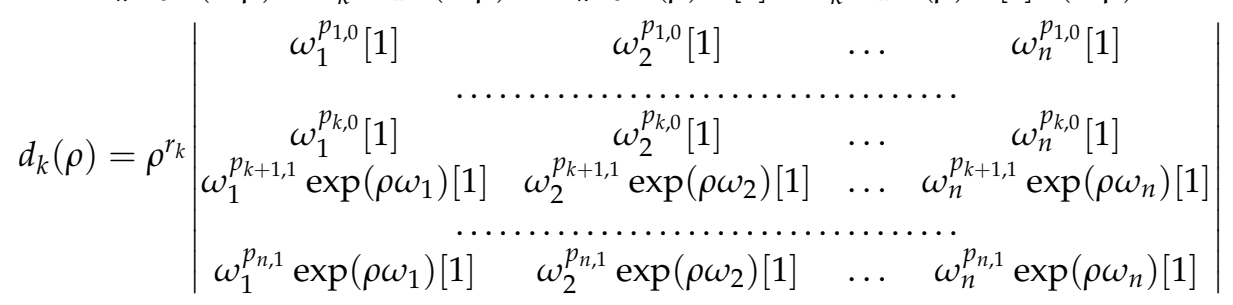

as $|\rho| \rightarrow \infty, \rho \in \bar{\Gamma}$. In view of (22), we arrive at the asymptotics 


$$
d_{k}(\rho)=\rho^{r_{k}} \operatorname{det}\left(T_{k} P_{0} \Omega+T_{k}^{\perp}\right) \operatorname{det}\left(T_{k}+T_{k}^{\perp} P_{1} \Omega\right) \exp \left(\rho s_{k}\right)[1],
$$

as $|\rho| \rightarrow \infty, \arg \rho=\varphi$. It can be shown that the determinants in (42) are non-zero. Therefore, substituting (39) and (42) into (41), we arrive at (37) for $j=k$.

According to the definition of $\Delta_{j k}(\lambda)$ and Theorem 1, if we replace the row $U_{k 0}$ by $U_{j 0}$, we obtain $\Delta_{j k}(\lambda)$ instead of $\Delta_{k k}(\lambda)$ for $k<j$. Therefore, the asymptotic formulas for $\Delta_{j k}(\lambda)$, $k<j$ can be derived similarly to the ones for $\Delta_{k k}(\lambda)$ and have the form (37).

Remark 2. The asymptotics (37) implies $\Delta_{j k}(\lambda) \not \equiv 0$. It follows from the proof of Lemma 2 that, for each fixed $x \in[0,1]$, the entire matrix function $C(x, \lambda)$ has the order $\frac{1}{n}$ and so do the functions $\Delta_{j k}(\lambda)$.

Lemma 3. The following asymptotic relation holds as $|\rho| \rightarrow \infty, \arg \rho=\varphi$ :

$$
\Phi(x, \lambda)=\Theta(\rho) \Omega[I] E(x, \rho) \operatorname{diag}\left\{\rho^{-p_{k, 0}} b_{k k}^{0}\right\}_{k=1}^{n},
$$

where $x \in[0,1)$ is fixed, $\lambda=\rho^{n}, b_{k k}^{0}$ are non-zero constants.

Proof. Fix a sector $\Gamma$ and expand the columns $\vec{\Phi}_{k}(x, \lambda)$ with respect to the columns of the matrix $Y(x, \rho)=\left[\vec{y}_{k}(x, \rho)\right]_{k=1}^{n}$ :

$$
\vec{\Phi}_{k}(x, \lambda)=Y(x, \rho) B_{k}(\rho), \quad k=\overline{1, n},
$$

where $\lambda=\rho^{n}, \rho \in \bar{\Gamma},|\rho| \geq \rho^{*}, B_{k}(\rho)=\operatorname{col}\left(b_{1 k}(\rho), b_{2 k}(\rho), \ldots, b_{n k}(\rho)\right)$. Clearly, the boundary conditions (27) can be rewritten in the form

$$
T_{k} U_{0} \vec{\Phi}_{k}(0, \lambda)+T_{k}^{\perp} U_{1} \vec{\Phi}_{k}(1, \lambda)=e_{k}, \quad k=\overline{1, n},
$$

where $e_{k}$ is the $k$-th column of the unit matrix $I$. Consequently, we obtain the linear system with respect to the vector $B_{k}(\rho)$ for each fixed $k$ :

$$
\left(T_{k} U_{0} Y(0, \rho)+T_{k}^{\perp} U_{1} Y(1, \rho)\right) B_{k}(\rho)=e_{k} .
$$

Obviously, the determinant of this system equals $d_{k}(\rho)$ from the proof of Lemma 2. By Cramer's rule,

$$
b_{j k}(\rho)=\frac{d_{j k}(\rho)}{d_{k}(\rho)}, \quad j=\overline{1, n},
$$

where the determinant $d_{j k}(\rho)$ is obtained from $d_{k}(\rho)$ by the replacement of the $j$-th column by $e_{k}$. Analogously to (42), we derive

$$
\begin{aligned}
& d_{j k}(\rho)=-\rho^{r_{k}-p_{k, 0}} \operatorname{det}\left(T_{k-1} P_{0} \Omega P_{j k}+T_{k-1}^{\perp}\right) \operatorname{det}\left(T_{k}+T_{k}^{\perp} P_{1} \Omega\right) \exp \left(\rho s_{k}\right)[1], \quad j<k, \\
& d_{k k}(\rho)=\rho^{r_{k}-p_{k, 0}} \operatorname{det}\left(T_{k-1} P_{0} \Omega+T_{k-1}^{\perp}\right) \operatorname{det}\left(T_{k}+T_{k}^{\perp} P_{1} \Omega\right) \exp \left(\rho s_{k}\right)[1], \\
& d_{j k}(\rho)=-\rho^{r_{k}-p_{k, 0}} \operatorname{det}\left(T_{k-1} P_{0} \Omega+T_{k-1}^{\perp}\right) \operatorname{det}\left(T_{k}+T_{k}^{\perp} P_{1} \Omega P_{j k}\right) \exp \left(\rho\left(s_{k}+\omega_{k}-\omega_{j}\right)\right)[1], \quad j>k,
\end{aligned}
$$

$$
\begin{aligned}
& b_{j k}(\rho)=\rho^{-p_{k, 0}} b_{j k}^{0}[1], \quad j \leq k, \\
& b_{j k}(\rho)=O\left(\rho^{-p_{k, 0}} \exp \left(\rho\left(\omega_{k}-\omega_{j}\right)\right)\right), \quad j>k,
\end{aligned}
$$

where

$$
b_{j k}^{0}=-\frac{\operatorname{det}\left(T_{k-1} P_{0} \Omega P_{j k}+T_{k-1}^{\perp}\right)}{\operatorname{det}\left(T_{k} P_{0} \Omega+T_{k}^{\perp}\right)}, \quad j<k, \quad b_{k k}^{0}=\frac{\operatorname{det}\left(T_{k-1} P_{0} \Omega+T_{k-1}^{\perp}\right)}{\operatorname{det}\left(T_{k} P_{0} \Omega+T_{k}^{\perp}\right)} \neq 0 .
$$


The asymptotics for $b_{j k}(\rho)$ together with (23) and (44) yield

$$
\Phi_{k}^{[j]}(x, \lambda)=\rho^{-p_{k, 0}}\left(\rho \omega_{k}\right)^{j} b_{k k}^{0} \exp \left(\rho \omega_{k} x\right)[1],
$$

as $|\rho| \rightarrow \infty, \arg \rho=\varphi$, for each fixed $x \in[0,1), j=\overline{0, n-1}, k=\overline{1, n}$. In the matrix form, we obtain (43).

\section{Uniqueness Theorem}

In this section, we formulate and prove the uniqueness theorem for solution of Inverse Problem 4.1. First, we need an auxiliary lemma.

Lemma 4. Let $\left\{y_{k}(x)\right\}_{k=1}^{n}$ be arbitrary solutions of the equation $\ell_{n}(y)=\lambda y$ for a fixed $\lambda \in \mathbb{C}$, and let $Y(x)$ be the matrix function $\left[\vec{y}_{k}(x)\right]_{k=1}^{n}$. Then, $\operatorname{det} Y(x)$ is constant.

Proof. Clearly, the matrix function $Y(x)$ satisfies the Equation (18):

$$
Y^{\prime}(x)=(F(x)+\Lambda) Y(x) .
$$

The standard relation yields

$$
(\operatorname{det} Y(x))^{\prime}=\operatorname{trace}(F(x)+\Lambda) \operatorname{det} Y(x) .
$$

Obviously, trace $(F(x)+\Lambda)=$ trace $F(x)$. Formulas (6) and (7) for even $n$ and (9) for odd $n$ imply that trace $(F(x))=0$. Hence, $(\operatorname{det} Y(x))^{\prime}=0$, which yields the claim.

Along with $\Xi$, consider another collection $\tilde{\Xi}=\left\{\tilde{\tau}_{1}, \ldots, \tilde{\tau}_{m}, \tilde{\sigma}_{i_{n}}, \ldots, \tilde{\sigma}_{m-1}\right\}$ of the same class. We agree that, if a symbol $\gamma$ denotes an object related to $\Xi$, then the symbol $\tilde{\gamma}$ with tilde denotes the similar object related to $\tilde{\Xi}$. The coefficients $U_{0}$ and $U_{1}$ of the boundary conditions are supposed to be the same for $\Xi$ and $\tilde{\Xi}$. However, the quasi-derivatives of solutions $y$ and $\tilde{y}$ of the equations $\ell_{n}(y)=\lambda y$ and $\tilde{\ell}_{n}(\tilde{y})=\lambda \tilde{y}$, respectively, differ if $F(x) \neq \tilde{F}(x)$.

Theorem 2. If $M(\lambda) \equiv \tilde{M}(\lambda)$, then $\Xi=\tilde{\Xi}$, that is, $\tau_{k}(x)=\tilde{\tau}_{k}(x), \sigma_{k}(x)=\tilde{\sigma}_{k}(x)$ a.e. on $(0,1)$. Thus, the solution of Inverse Problem 4.1 is unique.

Proof. Define the $(n \times n)$ matrix of spectral mappings $\mathcal{P}(x, \lambda)=\left[\mathcal{P}_{j k}(x, \lambda)\right]_{j, k=1}^{n}$ as follows:

$$
\mathcal{P}(x, \lambda)=\Phi(x, \lambda)(\tilde{\Phi}(x, \lambda))^{-1} .
$$

Using the relations

$$
\Phi(x, \lambda)=C(x, \lambda) M(\lambda), \quad \tilde{\Phi}(x, \lambda)=\tilde{C}(x, \lambda) \tilde{M}(\lambda),
$$

and $M(\lambda) \equiv \tilde{M}(\lambda)$, we obtain

$$
\mathcal{P}(x, \lambda)=C(x, \lambda)(\tilde{C}(x, \lambda))^{-1} .
$$

It follows from the initial condition $U_{0} \tilde{C}(0, \lambda)=I$ and Lemma 4 that $\operatorname{det} \tilde{C}(0, \lambda)= \pm$ 1. Hence, the matrix function $(\tilde{C}(x, \lambda))^{-1}$ is entire in $\lambda$ for each fixed $x \in[0,1]$, similarly to $C(x, \lambda)$. Thus, $\mathcal{P}(x, \lambda)$ is also entire in $\lambda$.

On the other hand, using the asymptotics of Lemma 3 for $\Phi(x, \lambda)$ and $\tilde{\Phi}(x, \lambda)$, we derive

$$
\mathcal{P}(x, \lambda)=\Theta(\rho)[I](\Theta(\rho))^{-1},
$$

as $|\rho| \rightarrow \infty, \arg \rho=\varphi, \varphi \neq \frac{\pi k}{n}, \lambda=\rho^{n}, x \in[0,1)$. Hence, 


$$
\mathcal{P}_{j k}(x, \lambda)= \begin{cases}o(1), & j<k, \\ 1+o(1), & j=k, \\ o(\lambda), & j>k,\end{cases}
$$

as $|\lambda| \rightarrow \infty$ along any ray $\arg \lambda=\beta, \beta \notin\{0, \pi\}$, for each fixed $x \in[0,1)$. One can use Phragmen-Lindelöf's theorem (see [42]) and Liouville's theorem to show that $\mathcal{P}(x, \lambda)$ is a constant unit lower triangular matrix $\mathcal{P}(x)$ for each fixed $x \in[0,1)$.

Differentiating the relation

$$
\mathcal{P}(x) \tilde{\Phi}(x, \lambda)=\Phi(x, \lambda)
$$

and using (18) for $\Phi(x, \lambda)$ and $\tilde{\Phi}(x, \lambda)$ :

$$
\Phi^{\prime}(x, \lambda)=(F(x)+\Lambda) \Phi(x, \lambda), \quad \tilde{\Phi}^{\prime}(x, \lambda)=(\tilde{F}(x)+\Lambda) \tilde{\Phi}(x, \lambda),
$$

we obtain

$$
\mathcal{P}^{\prime}(x)+\mathcal{P}(x) \tilde{F}(x)=F(x) \mathcal{P}(x) .
$$

In view of (45) and the initial conditions

$$
C(0, \lambda)=\tilde{C}(0, \lambda)=U_{0}^{-1},
$$

we have $\mathcal{P}(0)=I$. Applying Lemma 1, we conclude that $F(x)=\tilde{F}(x)$ a.e. on $(0,1)$. This readily yields $\Xi=\tilde{\Xi}$.

Recall that, by using the spectra $\left\{\lambda_{s, j k}\right\}_{s>1}, 1 \leq k \leq j \leq n$, one can uniquely construct the Weyl matrix $M(\lambda)$ by formulas (35) and (32). Therefore, Theorem 2 implies the following corollary.

Corollary 1. If $\lambda_{s, j k}=\tilde{\lambda}_{s, j k}, s \geq 1,1 \leq k \leq j \leq n$, then $\Xi=\tilde{\Xi}$. Thus, the solution of Inverse Problem 4.2 is unique.

\section{Examples}

In this section, we provide the examples illustrating the main results of this paper for $n=2$ and $n=3$. Note that the considered operators are equivalent to the operators (4) and (5), arising in applications.

Example 1. $n=2$. In this case, $m=1$ and the differential expression (1) takes the form $l_{2}(y)=y^{\prime \prime}-\tau_{1}^{\prime}(x) y$. Thus, $\Xi=\left\{\tau_{1}\right\}, \tau_{1} \in L_{2}(0,1)$. The matrix $F=\mathscr{F}(\Xi)$ defined by (6) has the form

$$
F=\left[\begin{array}{cc}
\tau_{1} & 1 \\
-\tau_{1}^{2} & -\tau_{1}
\end{array}\right]
$$

The quasi-derivative is defined as $y^{[1]}=y^{\prime}-\tau_{1} y$, so Equation (17) takes the form

$$
\ell_{2}(y)=\left(y^{[1]}\right)^{\prime}+\tau_{1} y^{[1]}+\tau_{1}^{2} y=\lambda y, \quad x \in(0,1) .
$$

For each $a=0,1$, we have the two linear forms

$$
\mathcal{U}_{1 a}(y)=y(a), \quad \mathcal{U}_{2 a}=y^{[1]}(a)+h_{a} y(a) \quad\left(h_{a} \in \mathbb{C}\right)
$$

or vice versa. The Weyl matrix has the form

$$
M(\lambda)=\left[\begin{array}{cc}
1 & 0 \\
M_{21}(\lambda) & 1
\end{array}\right]
$$


That is, it has the only non-trivial element $M_{21}(\lambda)=-\frac{\Delta_{21}(\lambda)}{\Delta_{11}(\lambda)}$, which generalizes the Weyl function for the classical Sturm-Liouville operators (see $[14,15])$. By virtue of Theorem $1, \Delta_{11}(\lambda)$ and $\Delta_{21}(\lambda)$ are the characteristic functions of the eigenvalue problems for Equation (46) with the boundary conditions

$$
\mathcal{L}_{11}: \mathcal{U}_{10}(y)=\mathcal{U}_{21}(y)=0, \quad \mathcal{L}_{21}: \mathcal{U}_{20}(y)=\mathcal{U}_{21}(y)=0,
$$

respectively. Denote by $\left\{\lambda_{n}^{D D}\right\},\left\{\lambda_{n}^{R D}\right\},\left\{\lambda_{n}^{R D}\right\},\left\{\lambda_{n}^{R R}\right\}$ the eigenvalues of the boundary value problems for Equation (46) with the following boundary conditions:

$$
\begin{array}{lrr}
\left\{\lambda_{n}^{D D}\right\} \sim y(0)=y(1)=0, & \text { (Dirichlet-Dirichlet) } \\
\left\{\lambda_{n}^{R D}\right\} \sim y^{[1]}(0)+h_{0} y(0)=y(1)=0, & \text { (Robin-Dirichlet) } \\
\left\{\lambda_{n}^{D R}\right\} \sim y(0)=y^{[1]}(1)+h_{1} y(1)=0, & \text { (Dirichlet-Robin) } \\
\left\{\lambda_{n}^{R R}\right\} \sim y^{[1]}(0)+h_{0} y(0)=y^{[1]}(1)+h_{1} y(1)=0 . & \text { (Robin-Robin) }
\end{array}
$$

By virtue of Theorem 2, the Weyl function $M_{21}(\lambda)$ uniquely specifies the coefficient $\tau_{1}$. Depending on whether the boundary condition $\mathcal{U}_{21}(y)=0$ is the Dirichlet or the Robin one, the Weyl function $M_{21}(\lambda)$ is uniquely specified by the two spectra: either $\left\{\lambda_{n}^{D D}\right\},\left\{\lambda_{n}^{R D}\right\}$ or $\left\{\lambda_{n}^{D R}\right\}$, $\left\{\lambda_{n}^{R R}\right\}$. Hence, our results imply the following corollary.

Corollary 2. The coefficient $\tau_{1}$ of $\ell_{2}(y)$ is uniquely determined by either $\left\{\lambda_{n}^{D D}\right\},\left\{\lambda_{n}^{R D}\right\}$ or $\left\{\lambda_{n}^{D R}\right\},\left\{\lambda_{n}^{R R}\right\}$.

Note that the coefficients $h_{0}$ and $h_{1}$ are assumed to be known a priori, since they are not uniquely specified by the two spectra. This happens because the shift $\tau_{1} \rightarrow \tau_{1}+c, h_{j} \rightarrow h_{j}+c$, $j=0,1$, does not change the spectra. However, if $h_{j}$ is fixed for either $j=0$ or 1 , then one can uniquely recover $h_{1-j}$ together with $\tau_{1}$ from $\left\{\lambda_{n}^{D R}\right\},\left\{\lambda_{n}^{R R}\right\}$. These results coincide with the results of Hryniv and Mykytyuk [21].

Example 2. $n=3$. In this case, $m=1$ and

$$
\ell_{3}(y)=y^{(3)}+\left(\sigma_{0}(x) y\right)^{\prime}+\sigma_{0}(x) y+\mathrm{i} \tau_{1}^{\prime}(x) y .
$$

Thus, $\Xi=\left\{\tau_{1}, \sigma_{0}\right\}, \tau_{1}, \sigma_{0} \in L_{1}(0,1)$. The matrix $F=\mathscr{F}(\Xi)$ has the form

$$
F=\left[\begin{array}{ccc}
0 & 1 & 0 \\
-\left(\sigma_{0}+\mathrm{i} \tau_{1}\right) & 0 & 1 \\
0 & -\left(\sigma_{0}-\mathrm{i} \tau_{1}\right) & 0
\end{array}\right]
$$

The quasi-derivatives equal

$$
y^{[1]}=y^{\prime}, \quad y^{[2]}=y^{\prime \prime}+\left(\sigma_{0}+\mathrm{i} \tau_{1}\right) y, \quad y^{[3]}=\left(y^{[2]}\right)^{\prime}+\left(\sigma_{0}-\mathrm{i} \tau_{1}\right) y^{\prime},
$$

and $\ell_{3}(y)=y^{[3]}$. There are multiple possible choices of the linear forms $\mathcal{U}_{\xi a}(y)$. For instance, consider the following ones:

$$
\mathcal{U}_{\xi a}(y)=y^{[\xi-1]}(a), \quad \xi=\overline{1,3}, \quad a=0,1
$$

The Weyl matrix has the form

$$
M(\lambda)=\left[\begin{array}{ccc}
1 & 0 & 0 \\
M_{21}(\lambda) & 1 & 0 \\
M_{31}(\lambda) & M_{32}(\lambda) & 1
\end{array}\right]
$$


Our results imply that the coefficients $\tau_{1}$ and $\sigma_{0}$ are uniquely specified by the eigenvalues of five boundary value problems for equation $\ell_{3}(y)=\lambda y$ with the boundary conditions

$$
\begin{array}{ll}
\mathcal{L}_{11}: & y(0)=0, \quad y^{[1]}(1)=y^{[2]}(1)=0, \\
\mathcal{L}_{21}: & y^{[1]}(0)=0, \quad y^{[1]}(1)=y^{[2]}(1)=0, \\
\mathcal{L}_{31}: & y^{[2]}(0)=0, \quad y^{[1]}(1)=y^{[2]}(1)=0, \\
\mathcal{L}_{22}: & y(0)=y^{[1]}(0)=0, \quad y^{[2]}(1)=0, \\
\mathcal{L}_{32}: & y(0)=y^{[2]}(0)=0, \quad y^{[2]}(1)=0 .
\end{array}
$$

Funding: This work was supported by Grant 21-71-10001 of the Russian Science Foundation.

Conflicts of Interest: The author declares no conflict of interest.

\section{References}

1. Mirzoev, K.A.; Shkalikov, A.A. Differential operators of even order with distribution coefficients. Math. Notes 2016, 99 , 779-784. [CrossRef]

2. Mirzoev, K.A.; Shkalikov, A.A. Ordinary differential operators of odd order with distribution coefficients. arXiv 2019, arXiv:1912.03660.

3. Naimark, M.A. Linear Differential Operators, 1st ed.; Parts I, II; Ungar: New York, NY, USA, 1967, 1968.

4. Deift, P.; Tomei, C.; Trubowitz, E. Inverse scattering and the Boussinesq equation. Comm. Pure Appl. Math. 1982, 35, 567-628. [CrossRef]

5. McKean, H. Boussinesq's equation on the circle. Comm. Pure Appl. Math. 1981, 34, 599-691. [CrossRef]

6. Braeutigam, I.N.; Polyakov, D.M. On the asymptotics of eigenvalues of a third-order differential operator. St. Petersburg Math. J. 2020, 31, 585-606. [CrossRef]

7. Badanin, A.V.; Korotyaev, E.L. Spectral asymptotics for the third order operator with periodic coefficients. J. Diff. Equ. 2012, 253, 3113-3146. [CrossRef]

8. Badanin, A.; Korotyaev, E.L. Third-order operators with three-point conditions associated with Boussinesq's equation. Appl. Anal. 2021, 100, 527-560. [CrossRef]

9. Timoshenko, S.P.; Woinowsky-Krieger, S. Theory of Plates and Shells; McGraw-Hill: New York, NY, USA, 1959.

10. Badanin, A.; Korotyaev, E.L. Resonances for Euler-Bernoulli operator on the half-line. J. Diff. Equ. 2017, 263, 534-566. [CrossRef]

11. Mikhlin, S.G. Variational Methods in Mathematical Physics; Nauka: Moscow, Russia; Pergamon: Oxford, UK, 1964.

12. Yakubovich, V.A.; Starzhinskii, V.M. Linear Differential Equations with Periodic Coeffcients and Their Applications; Nauka: Moscow, Russia, 1972.

13. Polyakov, D.M. Spectral estimates for the fourth-order operator with matrix coefficients. Comp. Math. Math. Phys. 2020, 60, 1163-1184. [CrossRef]

14. Freiling, G.; Yurko, V. Inverse Sturm-Liouville Problems and Their Applications; Nova Science Publishers: Huntington, NY, USA, 2001.

15. Marchenko, V.A. Sturm-Liouville Operators and Their Applications; Birkhauser: Boston, MA, USA, 1986.

16. Levitan, B.M. Inverse Sturm-Liouville Problems; VNU Sci. Press: Utrecht, The Netherlands, 1987.

17. Pöschel, J.; Trubowitz, E. Inverse Spectral Theory; Academic Press: New York, NY, USA, 1987.

18. Albeverio, S.; Gesztesy, F.; Hoegh-Krohn, R.; Holden, H. Solvable Models in Quantum Mechanics, 2nd ed.; AMS Chelsea Publishing: Providnce, RI, USA, 2005.

19. Savchuk, A.M.; Shkalikov, A.A. Sturm-Liouville operators with distribution potentials. Trans. Moscow Math. Soc. 2003, 64, 143-192.

20. Hryniv, R.O.; Mykytyuk, Y.V. Inverse spectral problems for Sturm-Liouville operators with singular potentials. Inverse Probl. 2003, 19, 665-684. [CrossRef]

21. Hryniv, R.O.; Mykytyuk, Y.V. Inverse spectral problems for Sturm-Liouville operators with singular potentials. II. Reconstruction by two spectra. North-Holl. Math. Stud. 2004, 197, 97-114.

22. Hryniv, R.O.; Mykytyuk, Y.V. Half-inverse spectral problems for Sturm-Liouville operators with singular potentials. Inverse Probl. 2004, 20, 1423-1444. [CrossRef]

23. Hryniv, R.O.; Mykytyuk, Y.V. Inverse spectral problems for Sturm-Liouville operators with singular potentials. IV. Potentials in the Sobolev space scale. Proc. Edinburgh Math. Soc. 2006, 49, 309-329. [CrossRef]

24. Savchuk, A.M.; Shkalikov, A.A. Inverse problem for Sturm-Liouville operators with distribution potentials: Reconstruction from two spectra. Russ. J. Math. Phys. 2005, 12, 507-514.

25. Savchuk, A.M.; Shkalikov, A.A. Inverse problems for Sturm-Liouville operators with potentials in Sobolev spaces: Uniform stability. Funct. Anal. Appl. 2010, 44, 270-285. [CrossRef] 
26. Freiling, G.; Ignatiev, M.Y.; Yurko, V. A. An inverse spectral problem for Sturm-Liouville operators with singular potentials on star-type graph. Proc. Symp. Pure Math. 2008, 77, 397-408.

27. Mykytyuk, Y.V.; Trush, N.S. Inverse spectral problems for Sturm-Liouville operators with matrix-valued potentials. Inverse Probl. 2009, 26, 015009. [CrossRef]

28. Hryniv, R.; Pronska, N. Inverse spectral problems for energy-dependent Sturm-Liouville equations. Inverse Probl. 2012, $28,085008$. [CrossRef]

29. Pronska, N. Reconstruction of energy-dependent Sturm-Liouville equations from two spectra. Int. Equ. Oper. Theory 2013, 76, 403-419. [CrossRef]

30. Eckhardt, J.; Gesztesy, F.; Nichols, R.; Teschl, G. Supersymmetry and Schrödinger-type operators with distributional matrix-valued potentials. J. Spectr. Theory 2014, 4, 715-768. [CrossRef]

31. Guliyev, N.J. Schrödinger operators with distributional potentials and boundary conditions dependent on the eigenvalue parameter. J. Math. Phys. 2019, 60, 063501. [CrossRef]

32. Bondarenko, N.P. Solving an inverse problem for the Sturm-Liouville operator with singular potential by Yurko's method. Tamkang J. Math. 2021, 52, 125-154. [CrossRef]

33. Bondarenko, N.P. Direct and inverse problems for the matrix Sturm-Liouville operator with the general self-adjoint boundary conditions. Math. Notes 2021, 109, 358-378. [CrossRef]

34. Bondarenko, N.P. Inverse problem solution and spectral data characterization for the matrix Sturm-Liouville operator with singular potential. Anal. Math. Phys. 2021, 11, 145. [CrossRef]

35. Leibenson, Z.L. The inverse problem of spectral analysis for higher-order ordinary differential operators. Trudy Moskov. Mat. Obshch. 1966, 15, 70-144.

36. Leibenson, Z.L. Spectral expansions of transformations of systems of boundary value problems. Trudy Moskov. Mat. Obshch. 1971, $25,15-58$.

37. Yurko, V.A. Recovery of nonselfadjoint differential operators on the half-line from the Weyl matrix. Math. USSR-Sb. 1992, 72, 413-438. [CrossRef]

38. Yurko, V.A. Method of Spectral Mappings in the Inverse Problem Theory; Inverse and Ill-Posed Problems Series; VNU Science: Utrecht, The Netherlands, 2002.

39. Beals, R.; Deift, P.; Tomei, C. Direct and Inverse Scattering on the Line. In Mathematical Surveys and Monographs; AMS: Providence, RI, USA, 1988; Volume 28.

40. Savchuk, A.M.; Shkalikov, A.A. Asymptotic analysis of solutions of ordinary differential equations with distribution coefficients. Sb. Math. 2020, 211, 1623-1659. [CrossRef]

41. Rykhlov, V.S. Asymptotical formulas for solutions of linear differential systems of the first order. Results Math. 1999, 36, 342-353. [CrossRef]

42. Buterin, S.A.; Freiling, G.; Yurko, V. A. Lectures on the Theory of Entire Functions; Schriftenreihe der Fakultät für Mathematik, SM-UDE-779; Diusburg-Essen University: Duisburg, Germany, 2014; 37p. 